Research Article

\title{
Socially Responsible Investment Portfolio Construction with a Double-Screening Mechanism considering Machine Learning Prediction
}

\author{
Jun Zhang and Xuedong Chen \\ School of Management and Engineering, Capital University of Economics and Business, Beijing 100070, China \\ Correspondence should be addressed to Xuedong Chen; nmcxd@cueb.edu.cn
}

Received 3 September 2021; Accepted 26 October 2021; Published 17 November 2021

Academic Editor: Dehua Shen

Copyright (c) 2021 Jun Zhang and Xuedong Chen. This is an open access article distributed under the Creative Commons Attribution License, which permits unrestricted use, distribution, and reproduction in any medium, provided the original work is properly cited.

\begin{abstract}
Although socially responsible investment (SRI) has developed into an important investment style, only a small number of studies discuss SRI portfolio construction. In view of the overwhelming breakthrough of machine learning in prediction, this paper proposes SRI portfolio construction models by combining a double-screening mechanism considering machine learning prediction and an extended global minimum variance (GMV) model (or extended maximum Sharpe ratio (MSPR) model), which are, respectively, named double-screening socially responsible investment (DSSRI) portfolio models I and II. The proposed models consist of two stages, i.e., stock screening and asset allocation. First, this paper develops a novel double-screening mechanism incorporating environmental, social, and corporate governance (ESG) and return potential criteria to ensure that high-quality stocks with good ESG performance and high-return potential are input into the optimal portfolio. Specifically, to obtain accurate stock return predictions, an extreme learning machine model optimized by the genetic algorithm is employed to predict stock prices. Next, to trade off the financial and ESG objectives of SRI investors, an extended GMV model (or extended MSPR model) considering the ESG factor is introduced to determine the capital allocation proportion of the stocks. We take the A-share market of China as the sample to verify the effectiveness of the proposed models. The empirical results demonstrate that compared with alternative models, the proposed models can yield better annualized return and ESG score performance as well as competitive Sharpe ratio performance.
\end{abstract}

\section{Introduction}

Socially responsible investment (SRI) considers personal values and societal concerns in investment decisions $[1,2]$. SRI investors consider investing as an extension of their lifestyle and thus are willing to incorporate their social beliefs and values into financial activities [3]. With the increasing salience of social and environmental issues and the gradual awakening of investors' consciousness of environmental, social, and corporate governance (ESG) factors, SRI has presented rapid growth [4] and has become an important investment style. As of 2020, there were 3038 signatories in the global financial market agreeing to abide by the Principles for Responsible Investment supported by the United Nations, encompassing management assets of 103.4 trillion dollars. Compared with the levels in 2019, this number of signatories represented an increase of $20 \%$, and the management asset volume exhibited an increase of 666 trillion dollars (see Principles for Responsible Investment at http://www.unpri.org).

In fact, SRI has attracted wide attention from academia over the past decades. Researchers have explored SRI related topics from various perspectives, including the motivation of SRI investors $[5,6]$, socially responsible measurement $[7,8]$, and the relationship between ESG rating and finance returns of the firm [9, 10]. For more references, see [11]. However, only a small number of studies focuses on SRI portfolios. Unlike the conventional portfolio construction, SRI portfolio construction requires a stock screening process to ensure that the stocks in the portfolio are in line with SRI 
investors' social values. A few researchers have made valuable explorations of stock screening. For example, Auer [12] applied different screening methods to test the performance of SRI. The empirical results showed that compared with positive screening, which can damage investment performance, negative screenings with low cut-off rates are better. Kempf and Osthoff [13] compared the three SRI screening mechanisms of negative, positive, and best-in-class screening through an investment strategy of buying stocks with high social responsibility ratings and selling stocks with low social responsibility ratings. The best-in-class screening was proven to generally obtain the highest abnormal returns. Barnett and Salomon [14] evaluated the effect of the intensity of social screening on the financial performance of SRI funds. They concluded that the financial returns of the SRI funds decrease with increasing screening intensity, while a rebound in financial returns occurs once the screening intensity reaches a maximum. It is worth mentioning that the stock screening conditions in previous studies were based solely on ESG criteria. However, as discussed by Diouf et al. [15], MacKenzie and Lewis [16], and Rosen et al. [17], SRI investors are concerned with both ESG and financial performance at the same time. There is no conclusive evidence that stocks screened based on ESG criteria can deliver high returns $[2,18-20]$. Therefore, it is necessary to consider the return potential of candidate stocks in the screening process to ensure that the screened stocks meet both the ESG criteria and the return demand of SRI investors.

To screen the potential high-return stocks, reliable stock return prediction is critical. Machine learning methods with excellent learning capability have been widely used for stock return prediction. The representative machine learning models for stock prediction include support vector machine regression (SVR) [21, 22], artificial neural networks (ANNs) $[23,24]$, fuzzy logic system $[25,26]$, deep neural network (DNN) [27, 28], convolutional neural network [29, 30], long short-term memory model (LSTM) [31-33], and ensemble learning [34, 35]. Recently, extreme learning machine (ELM), which is a novel machine learning model, has received attention due to its powerful performance. For instance, Lie et al. [36] exploited the high accuracy and quick learning advantages of ELM to predict the stock price and then generate trading signals. Dash and Dash [37] employed ELM to train a computational efficient functional link artificial neural network and obtained better prediction effects for stock index movements than those with the backpropagation (BP) algorithm. Wei et al. [38] effectively improved the accuracy of exchange rate forecasting using a hybrid model of kernel-based ELM. Jiang et al. [39] developed two hybrid models for stock price prediction that employed ELM as the predictor. The empirical results showed that the proposed models had high prediction accuracy. Although ELM is capable of achieving good prediction accuracy, its main drawback is the mechanism of randomly generating input weights and biases of the hidden layer, which may lead to inferior solutions and thus undermine its stability and performance $[40,41]$. To overcome this drawback, an effective strategy is to introduce an intelligent algorithm to optimize the input weights and biases of the hidden layer of ELM
[40, 42, 43]. The genetic algorithm (GA), a classic and excellent intelligent algorithm, has been utilized to optimize machine learning models, i.e., ELM [44-46] and BP [47]. In particular, ELM optimized by the GA (GA-ELM) has been successfully applied in various prediction topics, such as $\mathrm{CO}_{2}$ emission [44], wind power [45], and gas concentration [46]. Therefore, this paper introduces the GA-ELM to predict stock prices and then screens stocks with high-return potential based on the prediction results.

Once the stock screening is completed, SRI investors next need to determine the optimal investment weight of screened stocks. The mean-variance (MV) model, as the basis of modern portfolio theory, is still a common choice for SRI portfolio optimization. On this topic, only a handful of scholars have done relevant research. For example, Oikonomou et al. [48] tried to explore optimal ways to build SRI portfolios. They applied six models (three simplistic and three sophisticated) to construct SRI portfolios and compared the performance of each. The results indicated that the SRI portfolios based on the sophisticated optimization models represented by MV had superior risk-return trade-offs relative to the simplistic models (e.g., those with naive diversification). Branch et al. [49] analysed the performance of quantitative methodologies in ESG portfolio construction. They concluded that quantitative methods are effective at minimizing risk but could lead to unwanted exposure. Although the aforementioned studies performed meaningful and in-depth analyses, they either did not take the ESG factor into account in the portfolio optimization process or took ESG as a constraint condition instead of an optimization objective, thus failing to cater to investors' demand to maximize ESG. In [50], an extended MV was developed to construct an ESG portfolio, where minimizing the negative Sharpe ratio with the ESG score was taken as a novel variable in the objective function. The proposed model showed considerable superiority in ESG scores over the traditional MV model. However, this research lacked an effective screening process to guarantee that high-quality stocks with both high ESG and high-return potential were input into the SRI portfolio, leading to mediocre return performance. Similarly, several modified versions of MV models incorporating ESG objectives (see, for example, Utz et al. [51], Calvo et al. [52], Stephan et al. [53] and Bilbao et al. [54]) also fail to preselect high-quality stocks before SRI portfolio construction.

Based on the above analysis, this paper proposes two SRI portfolio construction models called double-screening socially responsible investment (DSSIR) portfolio models I and II (abbreviated as DSSIR-I and DSSIR-II), which combine a double-screening mechanism and portfolio optimization models. Specifically, the novel double-screening mechanism consists of ESG and return potential screening. First, in the ESG screening, stocks with inferior ESG scores are excluded from the investment universe to ensure that the screened stocks align with the social values of investors. Subsequently, in the return potential screening, to include stocks with high-return potential into the portfolio, the GA-ELM model is introduced to predict the price of the screened stock. Finally, the stocks with high ESG and high-return potential, 
as high-quality stocks, are input into the portfolio. After this stock screening, the portfolio optimization model is employed to weigh the screened high-quality stocks. In this work, to trade off the financial and ESG objectives of SRI investors, an extended global minimum variance (GMV) model (or extended maximum Sharpe ratio (MSPR) model) incorporating the ESG factor, which we call GMV-ESG (MSPR-ESG), is introduced as a portfolio optimization model. The main contributions of this paper are as follows:

(1) Unlike the majority of studies on SRI portfolio construction, this paper incorporates stock prediction into the portfolio selection, thereby providing a new method for SRI portfolio construction.

(2) This paper develops two novel SRI portfolio models called DSSRI-I and DSSRI-II, in which a doublescreening mechanism is designed to ensure highquality stocks (both high ESG and return potential) being the component of the SRI portfolio.

(3) The proposed models are compared with alternative models, including SSRI-GMV (or SSRI-MSPR), DSSRI-I (ELM) (or DSSRI-II (ELM)), DSSRI-I (SVR) (or DSSRI-II (SVR)), DSSRI-I (DNN) (or DSSRI-II (DNN)), and DSSRI-I (LSTM) (or DSSRIII (LSTM)). The comparisons demonstrate the feasibility and advantages of the proposed models.

The rest of this paper is as follows. In Section 2, we outline the related component models and introduce the proposed models. In Section 3, we describe datasets, parameter settings, and present and discuss the empirical results. In Section 4, we summarize the conclusions and prospects for future work.

\section{Methodology}

\subsection{GA-ELM Model}

2.1.1. Extreme Learning Machine. ELM, originally developed by Huang et al. [55], is an advanced algorithm for single-hidden layer feedforward neural network (SLFN), whose network topology consists of three layers, namely, the input layer, hidden layer, and output layer. The traditional algorithm for SLFN like BP algorithm determines the optimal weights through multiple iterations, while the ELM randomly generates input weights and biases of the hidden layer. The ELM can analytically calculate the output weights based on the minimum norm least squares solution. Therefore, compared with the BP algorithm, the ELM is capable of obtaining good generalized performance at an extremely fast speed. Given $Q$ sample pairs $\left(X_{i}, Y_{i}\right)$, where $i=1,2, \ldots, Q$, $X_{i}=\left[x_{1}, x_{2}, \ldots, x_{n}\right]^{T}$, and $Y_{i}=\left[y_{1}, y_{2}, \ldots, y_{m}\right]^{T}$, the following equation can be obtained for the ELM owning $L$ neurons in the hidden layer [55]:

$$
\sum_{i=1}^{L} \beta_{i} \varphi\left(w_{i} x_{j}+b_{i}\right)=y_{j}, \quad j=1, \ldots, Q,
$$

where $w_{i}=\left[w_{i 1}, w_{i 2}, \ldots, w_{i n}\right]^{T}$ denotes the input weight, $\beta_{i}=\left[\beta_{i 1}, \beta_{i 2}, \ldots, \beta_{i m}\right]^{T}$ represents the output weight, $\varphi(\cdot)$ is the activation function, and $b_{i}$ is the bias of the $i$ th neuron in the hidden layer. The matrix form of equation (1) is as follows [55]:

$$
H \beta=Y,
$$

where

$$
H=\left[\begin{array}{ccc}
\varphi\left(w_{1} x_{1}+b_{1}\right) & \cdots & \varphi\left(w_{L} x_{1}+b_{L}\right) \\
\vdots & \cdots & \vdots \\
\varphi\left(w_{1} x_{N}+b_{1}\right) & \cdots & \varphi\left(w_{L} x_{N}+b_{L}\right)
\end{array}\right]_{N \times L} .
$$

$H$ is the hidden layer output matrix. Accordingly, the output weight of the ELM can be obtained using the minimum norm least squares solution [55] as follows:

$$
\widehat{\beta}=H^{\dagger} T,
$$

where $H^{\dagger}$ represents the Moore-Penrose generalized inverse of $H$. The ELM can be implemented in the following four steps $[55,56]:$ (1) determine the number of neurons in the hidden layer; (2) randomly generate the input weights and biases of the hidden layer, $w_{i}$ and $b_{i}$; (3) compute the hidden layer output matrix, $H$; and (4) estimate the output weight, $\widehat{\beta}$.

2.1.2. Genetic Algorithm. The genetic algorithm is a classic intelligent algorithm first proposed by Holland in 1975 [57]. GA is regarded as a very efficient tool for optimization problems since it is computationally simple and powerful, as well as there are no restrictive assumptions about the solution space [58]. The core idea of the GA, inspired by the theory of 'survival of the fittest', is to imitate the biological evolution process of a population to obtain a globally optimal solution [59]. In solving a practical problem, the GA randomly generates a population of individuals (called chromosomes) as candidate solutions of a practical problem to evolve in successive generations, aiming to eliminate poor solutions within the population; thus, the remaining good solutions are further developed into better ones [60]. As a result, after finite iterations of evolution (until a predefined termination criterion is satisfied), an optimal solution from the population can be obtained. In the process of evolution, the chromosomes of each generation determine those in the next generation through three genetic operations, i.e., selection, crossover, and mutation.

(1) Selection Operation. This operation chooses chromosomes from the current population as parents to produce offspring input within the new population. A common selection technique is the route wheel method in which the probability of selection is proportionate to the fitness of each individual [61]. The fitness is the measure of the quality of the chromosome (solution), which is calculated by presetting the fitness function defined according to the practical problem. Given that $f\left(C^{i}\right)$ is the fitness of the $i$-th chromosome, the total fitness of the population of $G$ individuals can be expressed as follows [62]: 


$$
F=\sum_{i=1}^{G} f\left(C^{i}\right)
$$

The accumulated probability of each chromosome is as follows [62]:

$$
T_{g}=\frac{\sum_{i=1}^{g} f\left(C^{i}\right)}{F} .
$$

A random number $R \in[0,1]$ is generated, if $T_{g-1}<R<T_{g}$, individual $g$ is selected as the parent.

(2) Crossover Operation. A crossover operation that exchanges some parts of the two parents to produce a new pair of offspring is conducted to seek promising solutions from the search space [63]. Given a crossover probability $\varepsilon$, if generating a random number $R$ with a standard uniform distribution is less than $\varepsilon$, a locus is chosen between two neighbouring chromosomes, i.e., parents 1 and 2 . The chromosomes after this locus are exchanged, thereby forming new offsprings 1 and 2 [64]. The crossover processing can be expressed as follows:

$$
\left\{\begin{array} { l } 
{ \text { parent } ^ { 1 } = 1 0 0 1 1 0 1 1 1 } \\
{ \text { parent } ^ { 2 } = 1 1 1 0 0 0 0 1 0 }
\end{array} \Rightarrow \left\{\begin{array}{l}
\text { offspring }^{1}=10011 \mid 0010 \\
\text { offspring }^{2}=11100 \mid 0111
\end{array}\right.\right.
$$

(3) Mutation Operation. The mutation operation ensures the diversity of individuals and prevents solutions from being too similar to avoid obtaining the local optimal solution [65]. Similar to the crossover operation, given a mutation probability $\varepsilon$, if a random number $R$ with a standard uniform distribution is generated and is less than $\varepsilon$, a locus is chosen randomly on the parent, and the value of the locus is reversed, thereby producing a new offspring [64]. The mutation process can be expressed as follows:

$$
\text { parent }=100|\dot{1}| 10101 \Longrightarrow \text { offspring }=100|\dot{0}| 10101
$$

2.1.3. Optimization of the ELM Model Using the GA. Although the ELM has a fast learning speed and good generalization performance, the stability of the prediction results is weak because it randomly selects the input weights and the biases of the hidden neurons in training [40]. To improve the prediction performance of the ELM, the best input weights and the biases of the hidden layer should be determined to replace those from random selection. The most direct way to do so is by constructing a hybrid framework to combine an optimization algorithm and the ELM, thereby optimizing the input weights and the biases of the hidden layer randomly generated by the ELM [42]. Following previous studies [44-46], considering the sound performance in global search, GA is utilized to optimize the input weights and the biases of the hidden layer to improve the performance of the ELM in stock prediction. A detailed procedure of the GA-ELM algorithm is given in Algorithm 1.

2.2. Extended Portfolio Optimization Models. Markowitz's mean-variance (MV) model, as a classic portfolio optimization method, has been popular in the practice of financial investment. Based on a set of different return or risk levels, MV can determine the efficient frontier, at which the portfolios can obtain the maximum return for the given risk level or achieve the minimum risk for the given return level. There are two commonly known portfolio models on the efficient frontier, i.e., the GMV model [66] and the MSPR model [50]. The former denotes the minimum risk portfolio of the efficient frontier, and the latter denotes portfolio that maximizes the Sharpe ratio [66]. This paper incorporates the ESG factor into the objective functions of the GMV and MSPR models to form the GMV-ESG and MSPR-ESG models, respectively. The GMV-ESG model can be defined as follows:

$$
\begin{aligned}
& \min \sigma_{p}, \\
& \max \varphi_{p} \\
& \left\{\begin{array}{l}
\sigma_{p}=\sqrt{\sum_{i=1}^{n} \sum_{j=1}^{n} w_{i} \sigma_{i j} w_{j},} \\
\varphi_{p}=\sum_{i=1}^{n} w_{i} \varphi_{i}, \\
\sum_{i=1}^{n} w_{i}=1, \\
0 \leq w_{i} \leq 1, \quad \forall i=1,2, \ldots, n,
\end{array}\right.
\end{aligned}
$$

where $w_{i}$ is the weight of stock $i, \sigma_{i j}$ represents the covariance between stocks $i$ and $j$, and $\sigma_{p}$ is the standard deviation of the portfolio. Following the advice of Auer [12], considering that investors usually have only a vague understanding of ESG, a synthetic ESG score is used to quantify the ESG performance. $\varphi_{p}$ and $\varphi_{i}$ denote the expected ESG score of portfolio and stock $i$, respectively. For the MSPR-ESG model, similarly to the Sharpe ratio, we trade-off ESG score maximization and risk minimization, thereby incorporating the ESG score under a unit of risk into the MSPR model. The MSPR-ESG model can be defined as follows: 
Input: initialize the chromosome populations

Output: the optimal input weights and biases of the hidden layer of the ELM

(1) Begin

(2) Set the parameters of the ELM

(3) Define the fitness function

(4) Evaluate the fitness of all chromosomes in population

(5) $C=$ the best chromosome

(6) $g=1$

(7) while $g<$ maximum generation do

(8) for $i=0 \longrightarrow P$ do

(9) Generate $r d 1$ randomly in the interval $[0,1]$

(10) if $r d 1<$ crossover probability then

(11) Update the chromosome according to equation (7)

(12) end if

(13) Generate $r d 2$ randomly in the interval $[0,1]$

(14) if $r d 2<$ mutation probability then

(15) Update the chromosome according to equation (8)

(16) end if

(17) Evaluate the chromosome fitness

(18) end for

(19) Update the chromosome populations according to equation (6)

(20) Rank the chromosome populations according to fitness

(21) $C=$ the best current chromosome

(22) $g=g+1$

(23) end while

(24) Return the optimal input weights and biases of the hidden layer of the ELM according to C

(25) End

Algorithm 1: GA-ELM algorithm.

$$
\begin{aligned}
& \max \frac{u_{p}-r_{f}}{\sigma_{p}}, \\
& \max \frac{\varphi_{p}}{\sigma_{p}}, \\
& \left\{\begin{array}{l}
u_{p}=\sum_{i=1}^{n} w_{i} u_{i}, \\
\varphi_{p}=\sum_{i=1}^{n} w_{i} \varphi_{i}, \\
\sigma_{p}=\sqrt{\sum_{i=1}^{n} \sum_{j=1}^{n} w_{i} \sigma_{i j} w_{j},} \\
\sum_{i=1}^{n} w_{i}=1, \\
0 \leq w_{i} \leq 1, \quad \forall i=1,2, \ldots, n,
\end{array}\right.
\end{aligned}
$$

where $u_{i}$ and $u_{p}$ separately denote the expected return of stock $i$ and the portfolio, respectively, and $r_{f}$ denotes the risk-free return. In this study, the expected return $u_{i}$, covariance $\sigma_{i j}$, and ESG score $\varphi_{i}$ are obtained by the historical data.
2.3. The Proposed Models. The input of high-quality stocks plays an important role in constructing an optimal portfolio [67]. Thus, it is necessary to exclude inferior stocks that cannot satisfy investor demands from the investment universe. For most SRI investors, high-quality stocks not only need to meet ESG expectations but also have high-return potential. In the proposed models, a novel double-screening mechanism considering ESG and return potential is implemented to guarantee that high-quality stocks are input into the optimal portfolio. To be specific, low-ESG stocks are first cleaned from the investment universe based on the ESG threshold given by the investor. Subsequently, the GA-ELM model is utilized to predict the price of the remaining stocks in the future period, thereby obtaining the corresponding return prediction. Finally, the predicted returns are sorted in a descending order, and stocks with top-ranked returns are regarded as the ultimate high-quality stocks obtained from double-screening.

Furthermore, as Oikonomou et al. [48] claimed, in addition to optimizing the investment universe through the screening process, SRI investors also carefully consider portfolio selection techniques. The conventional GMV and MSPR models cannot trade off the financial and ESG performance of SRI investors since they do not incorporate the ESG factor. Therefore, this paper includes the ESG score within the objective functions of the GMV and MSPR models to form extended portfolio optimization models, i.e., the GMV-ESG and MSPR-ESG models, to balance the ESG 
and financial objectives of SRI investors. In summary, the proposed models are divided into the two stages of stock screening and asset allocation, involving four steps. The flowchart of the proposed models is shown in Figure 1, and a more detailed description of the proposed models is given as follows:

Step 1. Exclude low-ESG stocks. All sample stocks are ranked in descending order of ESG scores, i.e., $S=\left\{s_{1}, s_{2}, \ldots, s_{i}, s_{j}, \ldots, s_{n} \mid i<j, \operatorname{ESG}\left(s_{i}\right)>\operatorname{ESG}\left(s_{j}\right)\right\}$, where $s_{i}$ denotes stock $i$. Then, the ESG score threshold $\mathrm{ESG}_{T}$ is set, and stocks with ESG scores lower than the threshold $\mathrm{ESG}_{T}$ are excluded from $S$ to form a new stock pool $\bar{S}=\left\{s_{1}, s_{2}, \ldots, s_{i}, s_{j}, \ldots, s_{h} \mid i<j\right.$, ESG $\left.\left(s_{i}\right)>\operatorname{ESG}\left(s_{j}\right), 1<h<n, \operatorname{ESG}\left(s_{h}\right)>\mathrm{ESG}_{T}\right\}$.

Step 2. Predict stock prices. Because SRIs are usually medium-to long-term investments, we predict stock prices over a relatively long interval period instead of the next. First, stock price data are divided into a training set and a testing set, which are transformed into supervised learning forms. Let $P_{i}(t)$ denote the price of $s_{i} \in \bar{S}$ at time $t(t=1,2, \ldots, M), \Delta t$ denote the interval, and $\delta$ represent the time window size. Thus, the input matrix $X_{i}$ and output matrix $Y_{i}$ of $s_{i}$ can be expressed as follows:

$$
\begin{aligned}
X_{i} & =\left[\begin{array}{cccc}
P_{i}(1) & P_{i}(2) & \ldots & P_{i}(\delta) \\
P_{i}(2) & P_{i}(3) & \ldots & P_{i}(\delta+1) \\
\ldots & \ldots & \ldots & \ldots \\
P_{i}(M-\Delta t-\delta+1) & P_{i}(M-\Delta t-\delta+2) & \ldots & P_{i}(M-\Delta t)
\end{array}\right], \\
Y_{i} & =\left[\begin{array}{c}
P_{i}(\delta+\Delta t) \\
P_{i}(\delta+\Delta t+1) \\
\ldots \\
P_{i}(M)
\end{array}\right] .
\end{aligned}
$$

We stack $X_{i}(i=1,2, \ldots, h)$ in one large feature metric $\mathrm{V}$ with dimension $w \times \delta(w=h \times(M-\Delta t-\delta+1))$. Similarly, we can obtain one large target matrix $\mathrm{O}$ with dimension $w \times 1$. Subsequently, the feature metric $\mathrm{V}$ and target matrix $\mathrm{O}$ are fed to the GA-ELM model for training. Then, the optimal GA-ELM model obtained is applied to predict the prices of all stocks for the next $\Delta t$ periods, i.e., $\widehat{P}_{i}(t+\Delta t),(i=1, \ldots, h)$.

Step 3. Screen high-quality stocks. Converting predicted stock prices to predicted returns, $\widehat{R}_{s_{i}}(t+\Delta t)=\left(\widehat{P}_{i}(t+\Delta t)-P_{i}(t)\right) / P_{i}(t),(i=1, \ldots, h)$. Then, the obtained predicted returns $\widehat{R}_{s_{i}}(t+\Delta t)$ are sorted in descending order, and the top $k$ stocks are considered the ultimate high-quality stocks $\widehat{S}=\left\{s_{1}\right.$, $s_{2}, \ldots, s_{i}, s_{j}, \ldots, s_{k} \mid i<j, \widehat{R}_{s_{i}}(t+\Delta t)>\widehat{R}_{s_{j}}(t+\Delta t), \quad 1<$ $k<h\}$.

Step 4. Compute the proportion of capital allocation. The GMV-ESG and MSPR-ESG models are employed to determine the proportion of capital allocated to the high-quality stocks $\hat{S}$, thereby obtaining the optimal SRI portfolios. For convenience in solving, a multiobjective model is often transformed into a singleobjective model. Equations (9) and (10) can be expressed as follows:

$$
\begin{gathered}
\max \sum_{i=1}^{n} w_{i} \varphi_{i}-\sqrt{\sum_{i=1}^{n} \sum_{j=1}^{n} w_{i} \sigma_{i j} w_{j},} \\
\left\{\begin{array}{l}
\sum_{i=1}^{n} w_{i}=1, \\
0 \leq w_{i} \leq 1, \quad \forall i=1,2, \ldots, n, \\
\max \frac{u_{p}-r_{f}}{\sigma_{p}}+\frac{\varphi_{p}}{\sigma_{p}} \\
u_{p}=\sum_{i=1}^{n} w_{i} u_{i}, \\
\varphi_{p}=\sum_{i=1}^{n} w_{i} \varphi_{i}, \\
\sigma_{p}=\sqrt{\sum_{i=1}^{n} \sum_{j=1}^{n} w_{i} \sigma_{i j} w_{j}} \\
\sum_{i=1}^{n} w_{i}=1, \\
0 \leq w_{i} \leq 1, \quad \forall i=1,2, \ldots, n
\end{array}\right.
\end{gathered}
$$




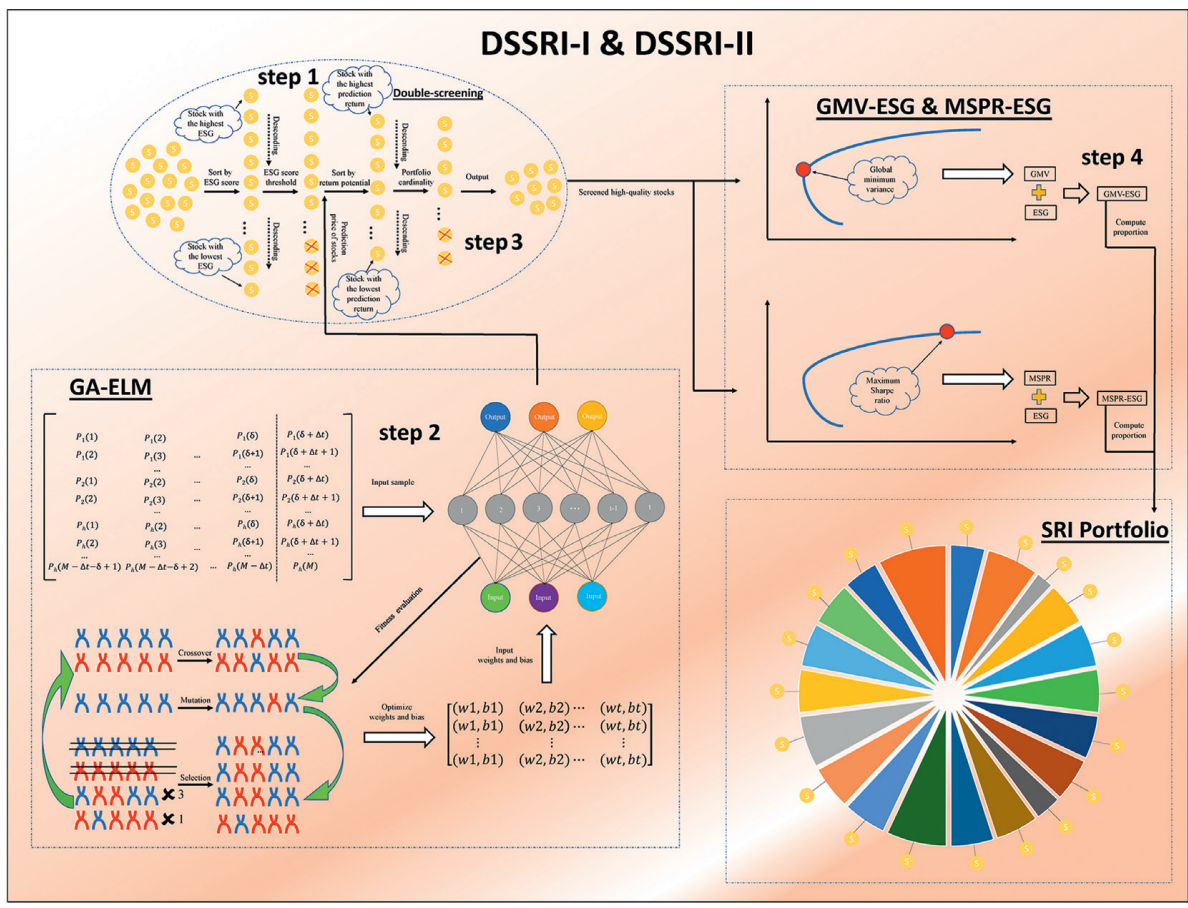

FIgURE 1: Flowchart of the proposed DSSRI-I and DSSRI-II.

\section{Empirical Results and Analysis}

3.1. Data Description. In this paper, we use the ESG score from Thomson Reuters ASSET 4, one of the most prominent professional ESG rating agencies. We obtain the monthly closing prices of stocks from January 1, 2010 to December 31, 2019 in Choice Database. At present, the Thomson Reuters ASSET 4 database reports the ESG scores of only a few Chinese listed companies. After excluding some companies whose ESG scores are much lower or whose stock price data are notably incomplete, we select 50 companies as the study samples, as shown in Table 1. The stock closing price time series is split into training and testing sets based on the yearly rolling window approach, thereby forming three study periods. Specifically, in the first study period, we apply the stock prices from 2010 to 2016 for the training set and from 2017 for the testing set. Then, we move to the next year in turn until the third study period, using the stock prices from 2012 to 2018 for the training set and the stock prices from 2019 for the testing set.

3.2. Parameter Settings. In our experiments, the interval $\Delta t$ is set to 3 and the time window size $\delta$ is set to 6 ; that is, the stock price from the first 6 months is used to predict the price 3 months ahead to achieve the quarterly prediction. The parameters of all relative models are presented in Table 2. The asset size of the portfolio is a critical factor to be considered by investors. Because inappropriate portfolio cardinality weakens the performance of the portfolio, it can either increase the fee or worsen the risk. Some studies provide us with valuable references on this subject; for example, Zulkifli et al. [68] argued that the inclusion of 15 stocks can diversify away the diversifiable risk. Tang [69] found that portfolios containing 20 stocks can eliminate $95 \%$ of the diversifiable risk. Considering the above conclusions and trading off the fee and risk, we set the portfolio cardinality $C=15,16,17,18,19$, and 20 . To guarantee the reliability of the prediction results, the ultimate prediction results of all models are obtained by computing the average value of 20 independent runs. The empirical study is implemented using Python 3.7 and TensorFlow 2.0 environments on a computer with $1.80 \mathrm{GHz} \mathrm{CPU}$ and 16.0 GB RAM.

3.3. Stock Double-Screening. In this section, doublescreening, including ESG screening and return potential screening, is performed. First, considering the limited stock universe in this study, we censor the $20 \%$ of stocks with the lowest ESG scores to complete the ESG screening. Next, for the remaining stocks, we use the GA-ELM model to predict the stock prices, further screening out stocks based on their return potential. To demonstrate the performance of the GA-ELM in terms of stock screening, we compare it with four advanced machine learning models: ELM, SVR, DNN, and LSTM. For prediction evaluation, several statistical criteria, such as the mean square error, root mean square error, and mean absolute error, are commonly used to measure the error of the prediction model. However, considering the purpose of the quantitative investment, these statistical metrics may not truly meet the financial goals of the investor. As mentioned in [70], practitioners focus more on potential trading profitability than on the prediction accuracy of models. A straightforward fact is that the more high-return stocks a portfolio contains, the greater is its probability of yielding high returns. Therefore, in this paper, 
TABLE 1: The stock exchange codes of the selected companies.

\begin{tabular}{ccccc}
\hline $000002 . S Z$ & $000039 . S Z$ & $000063 . S Z$ & $000157 . S Z$ & $000338 . S Z$ \\
\hline $000776 . S Z$ & $000898 . S Z$ & $002142 . S Z$ & $002594 . S Z$ & $600011 . S H$ \\
$600016 . S H$ & $600026 . S H$ & $600028 . S H$ & $600029 . S H$ & $600030 . S H$ \\
$600036 . S H$ & $600188 . S H$ & $600196 . S H$ & $600362 . S H$ & $600377 . S H$ \\
$600600 . S H$ & $600808 . S H$ & $600875 . S H$ & $601088 . S H$ & $601111 . S H$ \\
$601186 . S H$ & $601238 . S H$ & $601288 . S H$ & $601318 . S H$ & $601328 . S H$ \\
$601336 . S H$ & $601390 . S H$ & $601398 . S H$ & $601600 . S H$ & $601607 . S H$ \\
$601618 . S H$ & $601628 . S H$ & $601727 . S H$ & $601766 . S H$ & $601800 . S H$ \\
$601808 . S H$ & $601857 . S H$ & $601866 . S H$ & $601898 . S H$ & $601899 . S H$ \\
$601919 . S H$ & $601939 . S H$ & $601988 . S H$ & $601991 . S H$ & $601998 . S H$ \\
\hline
\end{tabular}

we screen high-return stocks to evaluate the prediction models. In other words, a good prediction model for quantitative investment can screen more high-return stocks than an inferior prediction model can. Obviously, there are two extreme cases: the best model can screen all high-return stocks, and the worst model cannot screen any.

The top $N$ stocks with the highest return among candidate stocks are regarded as high-return stocks. To verify the accuracy and generalizable performance of the GA-ELM model, we can give $N$ different values. Considering that the value of $N$ directly determines the asset size of the SRI portfolio constructed, we set $N=15,16,17,18,19$, and 20 . Table 3 shows the minimum, maximum, mean, and average accuracy (average accuracy $=$ mean $/ N$ ) of the screened number of high-return stocks from 2017Q1 to 2019Q4 using different models. We find that the GA-ELM outperforms the other models in nearly all cases in terms of minimum, maximum, mean, and average accuracy. Specifically, when the number of high-return stocks $N=15,16,17,18,19$, and 20 , the values of minimum, maximum, mean, and average accuracy obtained by the GA-ELM are almost uniformly the highest. For example, when $N=15$, the GA-ELM can screen 6.25 high-return stocks on average, while the ELM screens 5.25. The average accuracy of the GA-ELM is $6.67 \%$ higher than that of the ELM. The comparison results imply that using GA to optimize ELM can effectively improve the performance of ELM. Furthermore, compared with conventional machine learning models, i.e., SVR, and deep learning methods, i.e., DNN and LSTM, the average number of high-return stocks obtained by GA-ELM separately increase by $0.58,1.17$, and 0.83 , and the corresponding average accuracy also improves by $3.89 \%, 7.78 \%$, and $5.56 \%$. Accordingly, the superiority of the GA-ELM is further confirmed. When the number of high-return stocks $N$ equals other values, i.e., $16,17,18,19$, or 20 , we obtain similar conclusions, which indicate that the GA-ELM model has good generalizability. Therefore, the GA-ELM is a promising tool for use in the practice of stock screening.

3.4. SRI Portfolio Construction. In this section, the screened high-quality stocks are input into the GMV-ESG and MSPRESG models to construct SRI portfolios. The proposed models are compared with several alternative models at different portfolio cardinalities along the dimensions of return (annualized return), risk-adjusted return (Sharpe ratio), and ESG (ESG score).
3.4.1. Outline of Alternative Models. To test the performance of the proposed models, two kinds of alternative models are introduced for comparison:

(1) Alternative Model I: SSRI-GMV (or SSRI-MSPR). These models represent conventional SRI strategies that do not consider the return potential of stocks in the screening stage and do not introduce the ESG factor into the portfolio optimization model. In these models, low-ESG stocks are first excluded using ESG screening. Next, to keep the portfolio cardinality consistent with that of the proposed models, the same number of stocks as in the proposed models are selected from the screened stocks. Then, the GMV model (or MSPR model) is utilized to construct the SRI portfolio.

(2) Alternative Model II: DSSRI-I (Based on Other Machine Learning Models) (or DSSRI-II (Based on Other Machine Learning Models)). The purpose of introducing these models is to prove that the screening results obtained by different machine learning prediction models can affect the performance of the SRI portfolio. Specifically, we first exclude some stocks with low-ESG scores from the investment universe. Subsequently, several advanced machine learning models, such as ELM, SVR, DNN, and LSTM, are employed separately to predict stock prices, thereby further screening stocks with high-return potential. Finally, as with the proposed models, the GMV-ESG model (or MSPR-ESG model) is utilized to construct the SRI portfolio.

\subsubsection{Alternative Model I Comparison}

(1) Comparison of the Proposed DSSRI-I and the SSRI-GMV. Table 4 reports the annualized return, Sharpe ratio, and ESG score of the proposed DSSRI-I and the SSRI-GMV at different portfolio cardinalities $(C=15,16,17,18,19$, and 20). The performance comparison results of the two models are shown in Figure 2. It is obvious that the proposed DSSRI-I provides the highest values on the three evaluation metrics. If we take portfolio cardinality $C=15$ as an example, the annualized return, Sharpe ratio, and ESG score of the SSRIGMV are $0.0444,0.3198$, and 55.3456, respectively. Correspondingly, these metrics for the proposed DSSRI-I are $0.0982,0.4930$, and 61.9809 , representing increases of $0.0538,0.1733$, and 6.6353 . For other portfolio cardinalities $(C=16,17,18,19$, and 20$)$, the proposed DSSRI-I still generate good performance on all evaluation metrics. Specifically, compared with the SSRI-GMV, the annualized return of the proposed DSSRI-I increases in the range of $0.0320-0.0428$, the Sharpe ratio increases in the range of $0.0800-0.1510$, and the ESG score increases in the range of 5.3184-6.3273. Furthermore, at the portfolio cardinality $C=17$, the proposed DSSRI-I provides the highest annualized return and Sharpe ratio of 0.1113 and 0.5517 , respectively. The highest ESG score is generated by the proposed model at 61.9809 at portfolio cardinality $C=15$. In summary, the proposed DSSRI-I can yield better ESG and financial performance than the SSRI-GMV. 
TABle 2: Parameter settings of different models.

\begin{tabular}{|c|c|c|}
\hline Prediction models & Parameter & Value \\
\hline SVR & $\begin{array}{c}\text { Kernel function } \\
\gamma \\
\mathrm{C}\end{array}$ & $\begin{array}{c}r b f \\
{\left[2^{-15}, 2^{-13}, \ldots, 2^{13}, 2^{15}\right]} \\
{\left[2^{-15}, 2^{-3}, \ldots, 2^{13}, 2^{15}\right]}\end{array}$ \\
\hline DNN & $\begin{array}{c}\text { Layer structure (number of neurons) } \\
\text { Activation function } \\
\text { Dropout } \\
\text { Learning rate } \\
\text { The number of epochs }\end{array}$ & $\begin{array}{c}\text { Dense (30) - Dense (3) - Dense (1) } \\
\text { Relu } \\
0.2 \\
0.01 \\
200\end{array}$ \\
\hline LSTM & $\begin{array}{c}\text { Layer structure (number of neurons) } \\
\text { Dropout } \\
\text { Learning rate } \\
\text { Number of epochs }\end{array}$ & $\begin{array}{c}\operatorname{LSTM}(60)-\text { Dense }(16)-\text { Dense }(1) \\
0.1 \\
0.01 \\
200\end{array}$ \\
\hline ELM & $\begin{array}{l}\text { Activation function } \\
\text { Neuron number of hidden layer }\end{array}$ & $\begin{array}{l}\text { Sigmoid } \\
100\end{array}$ \\
\hline GA & $\begin{array}{l}\text { Population size } \\
\text { Maximum generation } \\
\text { Crossover probability } \\
\text { Mutation probability }\end{array}$ & $\begin{array}{c}25 / 20 \\
100 / 500 \\
0.8 / 0.5 \\
0.01 / 0.01\end{array}$ \\
\hline
\end{tabular}

TABLE 3: Comparison of screening results obtained by different models.

\begin{tabular}{|c|c|c|c|c|c|c|c|c|}
\hline \multirow{2}{*}{ Model } & \multicolumn{4}{|c|}{ The number of high-return stock $=15$} & \multicolumn{4}{|c|}{ The number of high-return stock $=16$} \\
\hline & Minimum & Maximum & Mean & Average accuracy (\%) & Minimum & Maximum & Mean & Average accuracy (\%) \\
\hline SVR & 3.00 & 9.00 & 5.67 & 37.78 & 3.00 & 9.00 & 6.25 & 39.06 \\
\hline DNN & 3.00 & 7.00 & 5.08 & 33.89 & 3.00 & 8.00 & 5.83 & 36.46 \\
\hline LSTM & 3.00 & 9.00 & 5.42 & 36.11 & 3.00 & 10.00 & 5.83 & 36.46 \\
\hline ELM & 3.00 & 7.00 & 5.25 & 35.00 & 3.00 & 8.00 & 6.08 & 38.02 \\
\hline GA-ELM & 4.00 & 8.00 & 6.25 & 41.67 & 4.00 & 9.00 & 6.83 & 42.71 \\
\hline \multirow{2}{*}{ Model } & \multicolumn{4}{|c|}{ The number of high-return stock $=17$} & \multicolumn{4}{|c|}{ The number of high-return stock $=18$} \\
\hline & Minimum & Maximum & Mean & Average accuracy (\%) & Minimum & Maximum & Mean & Average accuracy (\%) \\
\hline SVR & 4.00 & 10.00 & 7.17 & 42.16 & 5.00 & 10.00 & 7.50 & 41.47 \\
\hline DNN & 3.00 & 10.00 & 6.75 & 39.71 & 4.00 & 12.00 & 7.92 & 43.98 \\
\hline LSTM & 3.00 & 10.00 & 6.58 & 38.73 & 4.00 & 11.00 & 7.67 & 42.59 \\
\hline ELM & 4.00 & 9.00 & 7.17 & 42.16 & 5.00 & 10.00 & 8.08 & 44.91 \\
\hline GA-ELM & 5.00 & 11.00 & 7.92 & 46.57 & 5.00 & 13.00 & 8.83 & 49.07 \\
\hline \multirow{2}{*}{ Model } & \multicolumn{4}{|c|}{ The number of high-return stock $=19$} & \multicolumn{4}{|c|}{ The number of high-return stock $=20$} \\
\hline & Minimum & Maximum & Mean & Average accuracy (\%) & Minimum & Maximum & Mean & Average accuracy (\%) \\
\hline SVR & 6.00 & 11.00 & 8.33 & 43.86 & 6.00 & 12.00 & 9.25 & 46.25 \\
\hline DNN & 5.00 & 13.00 & 8.75 & 46.05 & 7.00 & 14.00 & 9.50 & 47.50 \\
\hline LSTM & 6.00 & 12.00 & 8.67 & 45.61 & 7.00 & 13.00 & 9.50 & 47.50 \\
\hline ELM & 7.00 & 11.00 & 9.00 & 47.37 & 8.00 & 12.00 & 9.58 & 47.92 \\
\hline GA-ELM & 7.00 & 14.00 & 9.75 & 51.32 & 9.00 & 15.00 & 11.00 & 55.00 \\
\hline
\end{tabular}

The best values are demonstrated with bold font.

(2) Comparison of the Proposed DSSRI-II and the SSRI$M S P R$. The annualized return, Sharpe ratio, and ESG score of the two models are shown in Table 5. Figure 3 compares the performance of the two models. Obviously, DSSRI-II has higher evaluation metrics than the SSRI-MSPR at different portfolio cardinalities $(C=15,16,17,18,19$, and 20). For example, the annualized returns of the proposed DSSRI-II are $0.0882,0.0780,0.1057,0.0856,0.0905$, and 0.0887 at portfolio cardinalities $C=15$ to 20 , representing increases of $63.02 \%, 67.76 \%, 94.95 \%, 82.01 \%, 62.13 \%$, and $65.61 \%$ over those of the SSRI-MSPR. For the Sharpe ratio, the DSSRI-II performs similarly. Specifically, the best Sharpe ratio (0.5339) is obtained by the DSSRI-II with portfolio cardinality $C=17$. In contrast, the best Sharpe ratio of the SSRI-
MSPR is 0.3750 . When the portfolio cardinality $C=15$, the DSSRI-II generates the highest ESG score of 62.0703, which is $11.50 \%$ higher than the best score of the SSRI-MSPR, at 55.6684. In short, the proposed DSSRI-II outperforms the SSRI-MSPR in terms of annualized return, Sharpe ratio, and ESG score.

\subsubsection{Alternative Model II Comparison}

(1) Comparison of the Proposed DSSRI-I and the DSSRI-I (Based on Other Machine Learning Models). In this section, we contrast the proposed DSSRI-I with the DSSRI-I (ELM), DSSRI-I (SVR), DSSRI-I (DNN), and DSSRI-I (LSTM) to 
TABle 4: Comparison of the DSSRI-I and the SSRI-GMV.

\begin{tabular}{lcccccccrcr}
\hline \multirow{2}{*}{ Model } & \multicolumn{3}{c}{ Portfolio cardinality $C=15$} & \multicolumn{2}{c}{ Portfolio cardinality $C=16$} & \multicolumn{3}{c}{ Portfolio cardinality $C=17$} \\
& AR & SR & ESG & AR & SR & ESG & AR & SR & ESG \\
\hline DSSRI-I & $\mathbf{0 . 0 9 8 2}$ & $\mathbf{0 . 4 9 3 0}$ & $\mathbf{6 1 . 9 8 0 9}$ & $\mathbf{0 . 0 9 0 4}$ & $\mathbf{0 . 4 7 0 1}$ & $\mathbf{6 1 . 8 5 2 5}$ & $\mathbf{0 . 1 1 1 3}$ & $\mathbf{0 . 5 5 1 7}$ & $\mathbf{6 1 . 9 6 1 9}$ \\
SSRI-GMV & 0.0444 & 0.3198 & 55.3456 & 0.0583 & 0.3901 & 55.5345 & 0.0686 & 0.4281 & 55.6346 \\
\hline \multirow{2}{*}{ Model } & \multicolumn{2}{c}{ Portfolio } & cardinality & C & 18 & \multicolumn{2}{c}{ Portfolio cardinality } & C $=19$ & & \multicolumn{2}{c}{ Portfolio cardinality $C=20$} \\
& AR & SR & ESG & AR & SR & ESG & AR & SR & ESG \\
\hline DSSRI-I & $\mathbf{0 . 0 9 4 7}$ & $\mathbf{0 . 4 9 5 2}$ & $\mathbf{6 1 . 5 3 6 9}$ & $\mathbf{0 . 0 9 6 2}$ & $\mathbf{0 . 5 0 4 9}$ & $\mathbf{6 1 . 2 8 4 4}$ & $\mathbf{0 . 0 9 9 4}$ & $\mathbf{0 . 5 2 2 2}$ & $\mathbf{6 0 . 9 3 8 5}$ \\
SSRI-GMV & 0.0614 & 0.3931 & 55.5110 & 0.0564 & 0.3710 & 55.3652 & 0.0567 & 0.3712 & 55.6201 \\
\hline
\end{tabular}

$\mathrm{AR}=$ annualized return; $\mathrm{SR}=$ Sharpe ratio; $\mathrm{ESG}=\mathrm{ESG}$ score. The best values are demonstrated with bold font.

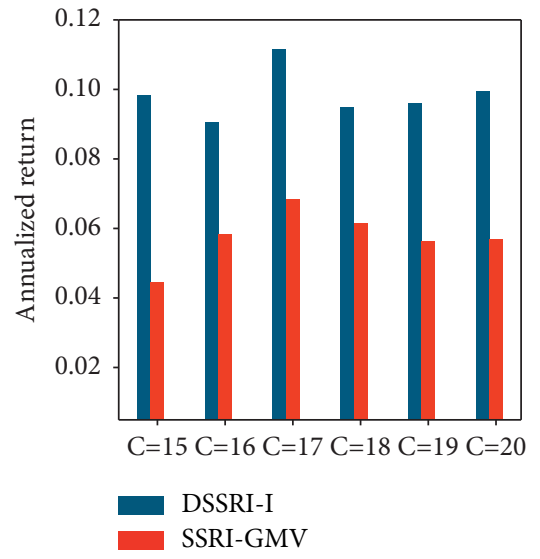

(a)

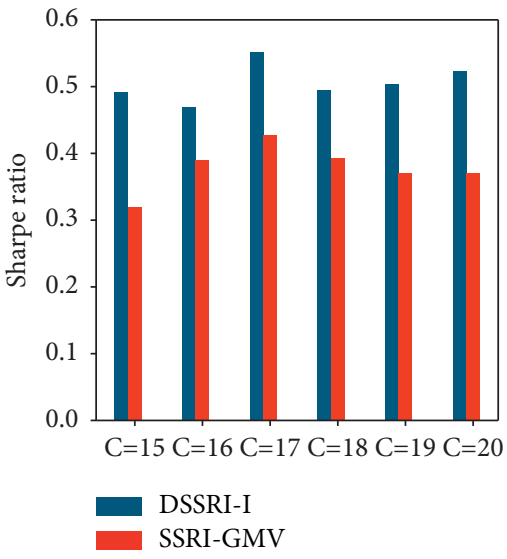

(b)

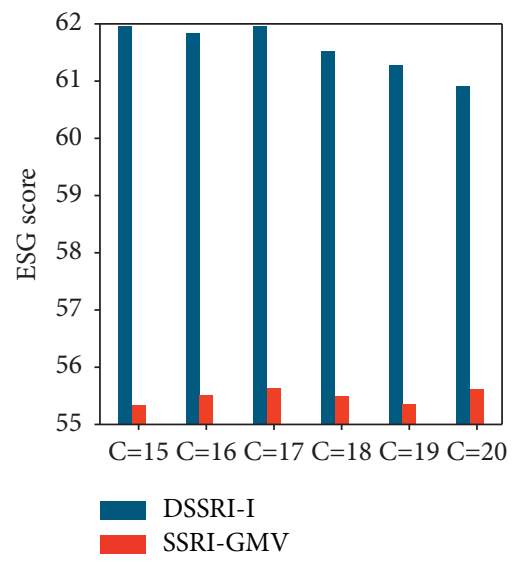

(c)

FIGURE 2: Performance of the DSSRI-I and the SSRI-GMV at different portfolio cardinalities.

TABLE 5: Comparison of the DSSRI-II and the SSRI-MSPR.

\begin{tabular}{lccccccrrr}
\hline \multirow{2}{*}{ Model } & \multicolumn{3}{c}{ Portfolio cardinality $C=15$} & \multicolumn{2}{c}{ Portfolio cardinality $C=16$} & \multicolumn{3}{c}{ Portfolio cardinality $C=17$} \\
& AR & SR & ESG & AR & SR & ESG & AR & SR & ESG \\
\hline DSSRI-II & $\mathbf{0 . 0 8 8 2}$ & $\mathbf{0 . 4 5 7 5}$ & $\mathbf{6 2 . 0 7 0 3}$ & $\mathbf{0 . 0 7 8 0}$ & $\mathbf{0 . 4 1 8 8}$ & $\mathbf{6 1 . 9 7 4 3}$ & $\mathbf{0 . 1 0 5 7}$ & $\mathbf{0 . 5 3 3 9}$ & $\mathbf{6 1 . 8 7 6 2}$ \\
SSRI-MSPR & 0.0541 & 0.3748 & 55.5466 & 0.0465 & 0.3311 & 55.4773 & 0.0542 & 0.3725 & 55.3819 \\
\hline \multirow{2}{*}{ Model } & \multicolumn{2}{c}{ Portfolio cardinality } & C $=18$ & \multicolumn{2}{c}{ Portfolio cardinality $C=19$} & \multicolumn{2}{c}{ Portfolio cardinality $C=20$} \\
& AR & SR & ESG & AR & SR & ESG & AR & SR & ESG \\
\hline DSSRI-II & $\mathbf{0 . 0 8 5 6}$ & $\mathbf{0 . 4 6 2 0}$ & $\mathbf{6 1 . 6 7 0 6}$ & $\mathbf{0 . 0 9 0 5}$ & $\mathbf{0 . 4 8 6 2}$ & $\mathbf{6 1 . 2 7 9 3}$ & $\mathbf{0 . 0 8 8 7}$ & $\mathbf{0 . 4 8 8 3}$ & $\mathbf{6 0 . 8 6 1 8}$ \\
SSRI-MSPR & 0.0470 & 0.3293 & 55.4834 & 0.0558 & 0.3750 & 55.6684 & 0.0536 & 0.3694 & 55.5192 \\
\hline
\end{tabular}

$\mathrm{AR}=$ annualized return; $\mathrm{SR}=$ Sharpe ratio; $\mathrm{ESG}=\mathrm{ESG}$ score. The best values are demonstrated with bold font.

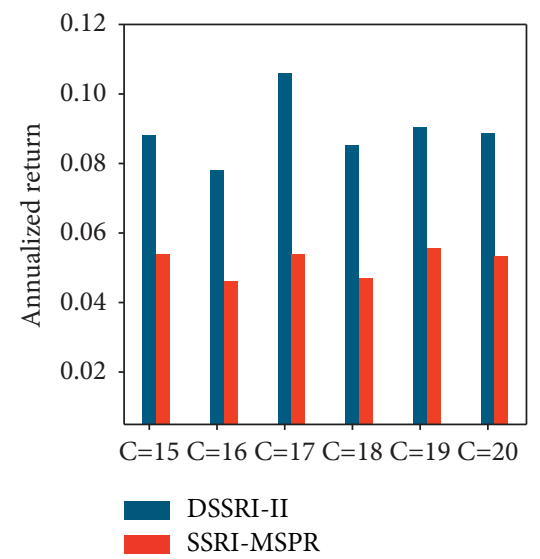

(a)

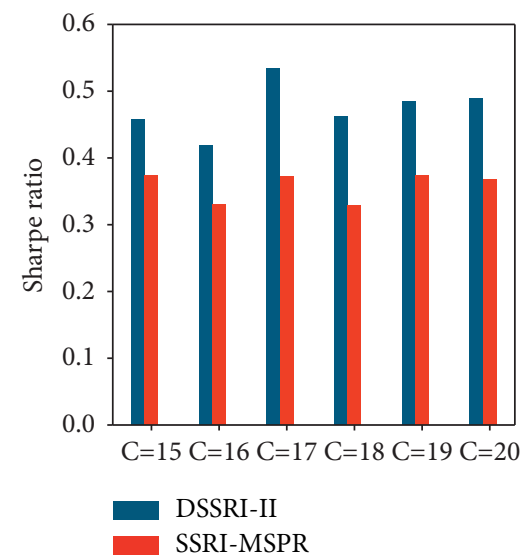

(b)

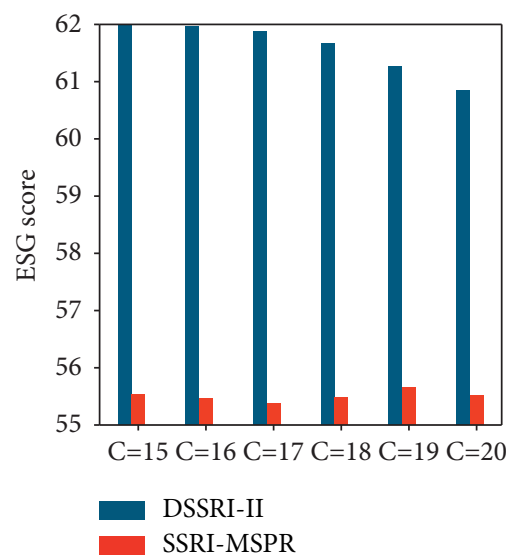

(c)

Figure 3: Performance of the DSSRI-II and the SSRI-MSPR at different portfolio cardinalities. 
TABLE 6: Comparison of the DSSRI-I model and the DSSRI-I (based on other machine learning models).

\begin{tabular}{|c|c|c|c|c|c|c|c|c|c|}
\hline \multirow{2}{*}{ Model } & \multicolumn{3}{|c|}{ Portfolio cardinality $C=15$} & \multicolumn{3}{|c|}{ Portfolio cardinality $C=16$} & \multicolumn{3}{|c|}{ Portfolio cardinality $C=17$} \\
\hline & AR & SR & ESG & $\mathrm{AR}$ & SR & ESG & $\mathrm{AR}$ & SR & ESG \\
\hline DSSRI-I & 0.0982 & 0.4930 & 61.9809 & 0.0904 & 0.4701 & 61.8525 & 0.1113 & 0.5517 & 61.9619 \\
\hline DSSRI-I (ELM) & 0.0274 & 0.2269 & 59.8493 & 0.0446 & 0.2969 & 59.4059 & 0.0525 & 0.3320 & 59.2332 \\
\hline DSSRI-I (SVR) & 0.0070 & 0.1304 & 58.6909 & 0.0098 & 0.1426 & 58.7900 & 0.0162 & 0.1741 & 58.6922 \\
\hline DSSRI-I (DNN) & 0.0901 & 0.5623 & 57.8888 & 0.0941 & 0.6063 & 57.5421 & 0.0705 & 0.4730 & 57.6859 \\
\hline DSSRI-I (LSTM) & 0.0455 & 0.3156 & 59.0560 & 0.0208 & 0.1949 & 58.9020 & 0.0260 & 0.2202 & 58.4004 \\
\hline \multirow{2}{*}{ Model } & \multicolumn{3}{|c|}{ Portfolio cardinality $C=18$} & \multicolumn{3}{|c|}{ Portfolio cardinality $C=19$} & \multicolumn{3}{|c|}{ Portfolio cardinality $C=20$} \\
\hline & $\mathrm{AR}$ & SR & ESG & AR & SR & ESG & $\mathrm{AR}$ & SR & ESG \\
\hline DSSRI-I & 0.0947 & 0.4952 & 61.5369 & 0.0962 & 0.5049 & 61.2844 & 0.0994 & 0.5222 & 60.9385 \\
\hline DSSRI-I (ELM) & 0.0498 & 0.3241 & 59.2538 & 0.0480 & 0.3193 & 59.0777 & 0.0537 & 0.3501 & 59.4691 \\
\hline DSSRI-I (SVR) & -0.0026 & 0.0765 & 58.6725 & 0.0052 & 0.1166 & 58.4987 & 0.0158 & 0.1711 & 58.5493 \\
\hline DSSRI-I (DNN) & 0.0688 & 0.4666 & 57.8780 & 0.0602 & 0.4293 & 57.6697 & 0.0567 & 0.4077 & 57.5114 \\
\hline DSSRI-I (LSTM) & 0.0388 & 0.2843 & 58.5475 & 0.0247 & 0.2145 & 58.4433 & 0.0567 & 0.3688 & 58.3013 \\
\hline
\end{tabular}

$\mathrm{AR}=$ annualized return; $\mathrm{SR}=$ Sharpe ratio; $\mathrm{ESG}=\mathrm{ESG}$ score. The best values are demonstrated with bold font.

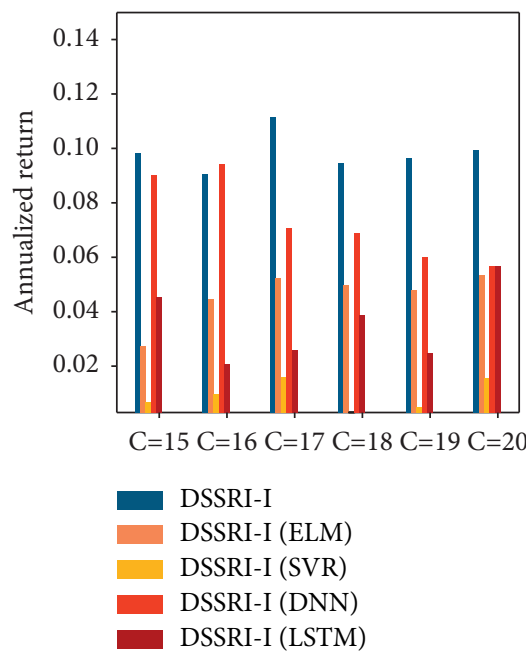

(a)

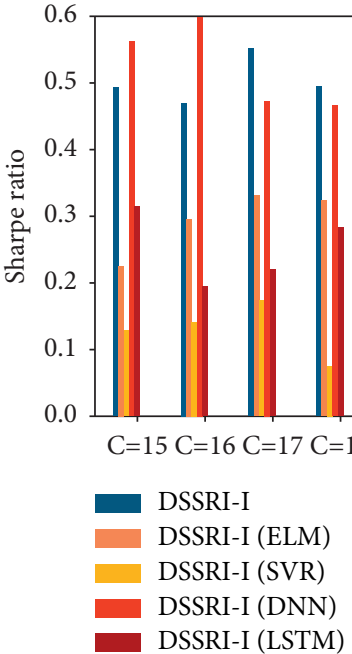

(b)
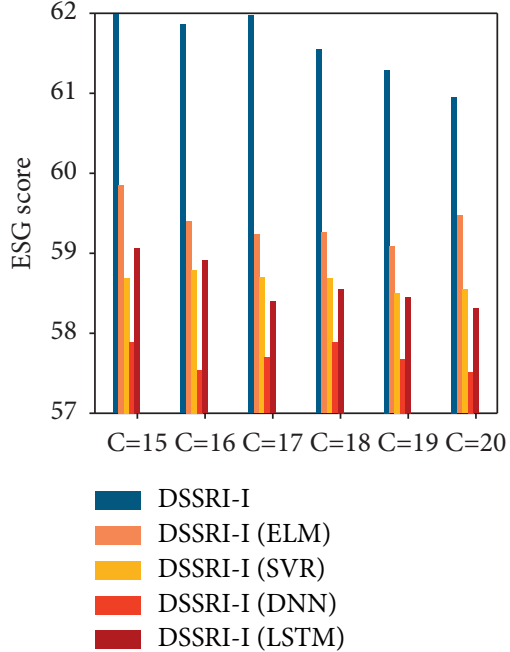

(c)

FIGURe 4: Performance of the DSSRI-I model and the DSSRI-I (based on other machine learning models) at different portfolio cardinalities.

verify the superiority of return potential screening based on the GA-ELM. The annualized return, Sharpe ratio, and ESG score of all models at different portfolio cardinalities $C=15$, $16,17,18,19$, and 20 are presented in Table 6 . The comparison of the evaluation metrics for the different models is displayed in Figure 4. First, we compare the proposed DSSRI-I with the DSSRI-I (ELM). As shown in Table 6 and Figure 4 , when the portfolio cardinality $C=15$, the annualized return of the proposed DSSRI-I is 0.0708 higher than that of the DSSRI-I (ELM). The Sharpe ratio of the proposed DSSRI-I is 0.4930 , which is $117.32 \%$ higher than that of the DSSRI-I (ELM). The highest ESG score (61.9809) is still obtained by the proposed DSSRI-I. For other portfolio cardinalities $(C=16,17,18,19$, and 20), similar conclusions are obtained. In general, the proposed model is superior to the DSSRI-I (ELM) in terms of annualized return, Sharpe ratio, and ESG score and thus proves again that GA optimization can improve the performance of the ELM. Subsequently, the proposed DSSRI-I is compared with SRI portfolio models based on double-screening considering other advanced machine learning models (SVR, DNN, and LSTM), i.e., DSSRI-I (SVR), DSSRI-I (DNN), and DSSRI-I (LSTM). From Table 6 and Figure 4, we can find that the highest annualized returns at almost all the given portfolio cardinality levels are obtained by the DSSRI-I. When the portfolio cardinalities are $C=17,18,19$ and 20, the proposed DSSRI-I provides the highest Sharpe ratio at 0.5517, 0.4952, 0.5049 , and 0.5222 , respectively. The proposed DSSRI-I generates the second-highest Sharpe ratio with portfolio cardinalities $C=15$ and 16, following the DSSRI-I (DNN). The DSSRI-I generates the highest ESG scores from portfolio cardinalities $C=15$ to 20 with values of $61.9809,61.8525$, $61.9619,61.5369,61.2844$, and 60.9385 , respectively. In general, compared with other SRI portfolio models based on advanced machine learning models, the proposed DSSRI-I presents obvious advantages in annualized return and ESG performance and has a competitive Sharpe ratio performance. Therefore, the proposed DSSRI-I based on the GAELM is capable of improving the ESG and financial performance of SRI portfolios. 
TABLE 7: Comparison of the DSSRI-II model and the DSSRI-II (based on other machine learning models).

\begin{tabular}{|c|c|c|c|c|c|c|c|c|c|}
\hline \multirow{2}{*}{ Model } & \multicolumn{3}{|c|}{ Portfolio cardinality $C=15$} & \multicolumn{3}{|c|}{ Portfolio cardinality $C=16$} & \multicolumn{3}{|c|}{ Portfolio cardinality $C=17$} \\
\hline & AR & SR & ESG & $\mathrm{AR}$ & SR & ESG & $\mathrm{AR}$ & SR & ESG \\
\hline DSSRI-II & 0.0882 & 0.4575 & 62.0703 & 0.0780 & 0.4188 & 61.9743 & 0.1057 & 0.5339 & 61.8762 \\
\hline DSSRI-II (ELM) & 0.0360 & 0.2659 & 59.8217 & 0.0458 & 0.3028 & 59.3619 & 0.0407 & 0.2845 & 59.1623 \\
\hline DSSRI-II (SVR) & 0.0110 & 0.1499 & 58.5464 & 0.0130 & 0.1580 & 58.9834 & 0.0212 & 0.1981 & 58.7566 \\
\hline DSSRI-II (DNN) & 0.0873 & 0.5465 & 57.8571 & 0.1024 & 0.6361 & 57.6394 & 0.0697 & 0.4616 & 57.7544 \\
\hline DSSRI-II (LSTM) & 0.0537 & 0.3505 & 59.1856 & 0.0298 & 0.2392 & 58.7573 & 0.0268 & 0.2240 & 58.6118 \\
\hline \multirow{2}{*}{ Model } & \multicolumn{3}{|c|}{ Portfolio cardinality $C=18$} & \multicolumn{3}{|c|}{ Portfolio cardinality $C=19$} & \multicolumn{3}{|c|}{ Portfolio cardinality $C=20$} \\
\hline & AR & SR & ESG & AR & SR & ESG & $\mathrm{AR}$ & SR & ESG \\
\hline DSSRI-II & 0.0856 & 0.4620 & 61.6706 & 0.0905 & 0.4862 & 61.2793 & 0.0887 & 0.4883 & 60.8618 \\
\hline DSSRI-II (ELM) & 0.0442 & 0.3002 & 59.2208 & 0.0444 & 0.3031 & 59.0595 & 0.0596 & 0.3728 & 59.4935 \\
\hline DSSRI-II (SVR) & 0.0019 & 0.1015 & 58.5811 & 0.0098 & 0.1399 & 58.5797 & 0.0162 & 0.1738 & 58.5194 \\
\hline DSSRI-II (DNN) & 0.0677 & 0.4653 & 57.8591 & 0.0698 & 0.4922 & 57.8490 & 0.0565 & 0.4030 & 57.4872 \\
\hline DSSRI-II (LSTM) & 0.0342 & 0.2612 & 58.6037 & 0.0306 & 0.2438 & 58.3080 & 0.0521 & 0.3441 & 58.4704 \\
\hline
\end{tabular}

$\mathrm{AR}=$ annualized return; $\mathrm{SR}=$ Sharpe ratio; $\mathrm{ESG}=\mathrm{ESG}$ score. The best values are demonstrated with bold font.

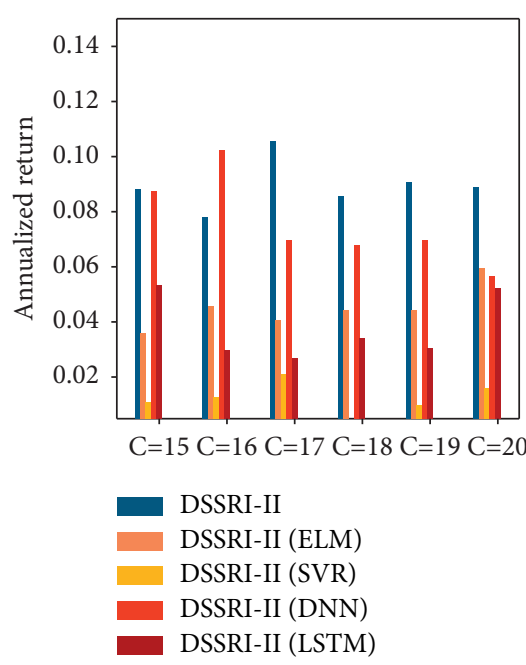

(a)

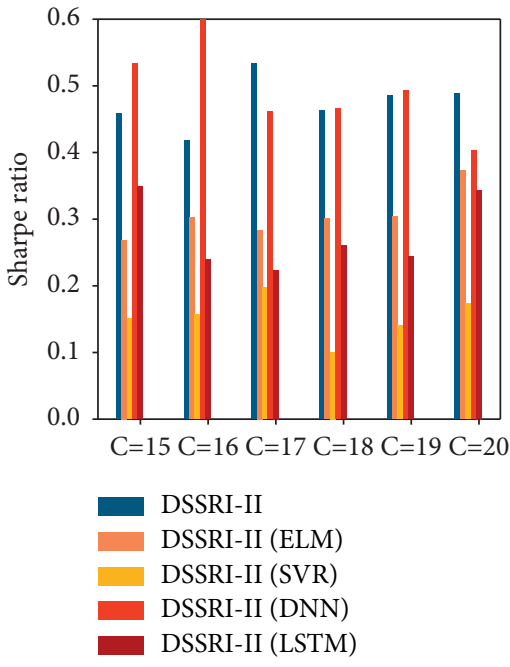

(b)

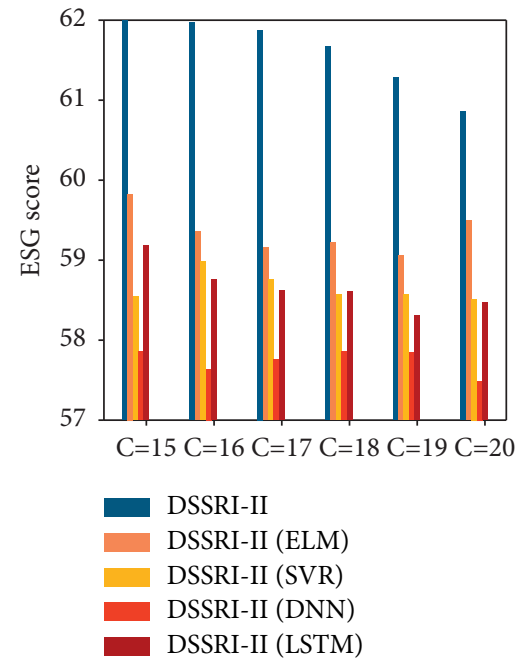

(c)

FIGURe 5: Performance of the DSSRI-II model and the DSSRI-II model (based on other machine learning models) at different portfolio cardinalities.

(2) Comparison of the Proposed DSSRI-II and the DSSRI-II (Based on Other Machine Learning Models). As shown in Table 7 and Figure 5, the proposed DSSRI-II performs well in terms of annualized return, Sharpe ratio, and ESG score. Specifically, for the annualized return, the proposed DSSRI-II obtains the highest values at almost all the given portfolio cardinalities. However, an exception is when the portfolio cardinality $C=16$, in which case the DSSRI-II (DNN) yields the highest annualized return of 0.1024 . The proposed DSSRI-II yields the second-highest annualized return with 0.0780 . The proposed DSSRI-II generates the second-highest Sharpe ratio at portfolio cardinalities $C=15,16,18$, and 19 and the highest Sharpe ratio at portfolio cardinalities $C=17$ and 20 . For the ESG score, with the portfolio cardinality $C=15$ taken as an example, the ESG score of the proposed model is 62.0703, representing an improvement of $3.76 \%, 6.02 \%, 7.28 \%$, and $4.87 \%$ over the DSSRI-II (ELM), DSSRI-II (SVR), DSSRIII (DNN), and DSSRI-II (LSTM), respectively. At the other portfolio cardinality levels, the proposed DSSRI-II is consistently ahead of the comparison models by at least $2.30 \%$ on the ESG score. In summary, similarly to the DSSRI-I, the DSSRI-II can provide good annualized return and ESG performance as well as competitive Sharpe ratio performance in comparison with the performance of the DSSRI-II (based on other machine learning models).

\section{Conclusion and Future Work}

In this work, we propose two SRI portfolio construction models called DSSRI-I and DSSRI-II based on machine learning, intelligent algorithm, and portfolio theory. The models consist of two stages: stock screening and asset allocation. In the stock screening stage, a doublescreening mechanism is developed to generate the input of the SRI portfolio. Specifically, low-ESG stocks are first excluded from the investment universe to guarantee that the 
assets are in line with the social values of SRI investors. Then, an ELM model with GA optimization is introduced to predict the prices of the remaining stocks and thus further screen high-return potential stocks. In the asset allocation stage, the extended GMV model (or extended MSPR model) considering the ESG factor is introduced to determine the investment proportion of the screened stocks from the first stage. To test the performance, we compared the proposed models with alternative models in terms of annualized return, Sharpe ratios, and ESG scores. The conclusions show the following: (1) compared with the conventional SSRIGMV (or SSRI-MSPR), the proposed DSSRI-I (or DSSRI-II) performs better on both ESG and financial measures. (2) The proposed DSSRI-I (or DSSRI-II) can obtain a higher annualized return and ESG score than the alternative models based on advanced machine learning models (i.e., DSSRI-I (ELM) (or DSSRI-II (ELM)), DSSRI-I (SVR) (or DSSRI-II (SVR)), DSSRI-I (DNN) (or DSSRI-II (DNN)), and DSSRI-I (LSTM) (or DSSRI-II (LSTM)). (3) The proposed models can generate a competitive Sharpe ratio performance in comparison with that of the above models. Therefore, considering the superior performance of the proposed models, they can be regarded as effective tools to construct SRI portfolios.

In the future, our work may focus on the following three aspects. First, other stock markets in different political, economic, and social environments should be studied to test the generalizability of the proposed models. Second, the performance of the proposed models can be further analysed by introducing various intelligent algorithms to optimize the ELM. Finally, the proposed models can be extended to a general framework for portfolio construction based on the personal preference of investors.

\section{Abbreviations}

ANNs: Artificial neural networks

BP: Back propagation

DNN: Deep neural network

DSSRI: Double-screening socially responsible investment

ELM: Extreme learning machine

ESG: Environmental, social, and corporate governance

GA: Genetic algorithm

GMV: Global minimum variance

LSTM: Long short-term memory

MSRP: Maximum Sharpe ratio

MV: $\quad$ Mean-variance

SLFN: Single-hidden layer feedforward neural network

SRI: $\quad$ Socially responsible investment

SVR: Support vector regression.

\section{Data Availability}

The data that support the findings of this study are available from the corresponding author upon reasonable request.

\section{Conflicts of Interest}

The authors declare that they have no conflicts of interest.

\section{Acknowledgments}

This work was supported by the Graduate Science and Technology Innovation Foundation of the Capital University of Economics and Business, China.

\section{References}

[1] Social Investment Forum Foundation, Report on Socially Responsible Investing Trends in the united states, Social Investment Forum Foundation, Washington, DC, 1999.

[2] M. Statman, "Socially responsible indexes," Journal of Portfolio Management, vol. 32, no. 3, pp. 100-109, 2006.

[3] K. Pattit, "Understanding socially responsible investing: the effect of decision frames and trade-off options," Journal of Business Ethics, vol. 87, no. 4, pp. 41-55, 2009.

[4] L. Renneboog, J. Horst, J. Ter Horst, and C. Zhang, "Socially responsible investments: institutional aspects, performance, and investor behavior," Journal of Banking \& Finance, vol. 32, no. 9, pp. 1723-1742, 2008.

[5] D. J. Beal, M. Goyen, and P. Philips, "Why do we invest ethically?” Journal of Investing, vol. 14, no. 3, pp. 66-78, 2005.

[6] W. R. Pasewark and M. E. Riley, "It's a matter of principle: the role of personal values in investment decisions," Journal of Business Ethics, vol. 93, no. 2, pp. 237-253, 2010.

[7] T. A. Hart and M. Sharfman, "Assessing the concurrent validity of the revised kinder, lydenberg, and domini corporate social performance indicators," Business \& Society, vol. 54, no. 5, pp. 575-598, 2015.

[8] L. Widyawati, "Measurement concerns and agreement of environmental social governance ratings," Accounting and Finance, vol. 61, no. S1, pp. 1589-1623, 2021.

[9] N. Sachin and R. Rajesh, "An empirical study of supply chain sustainability with financial performances of indian firms," Environment Development and Sustainability, vol. 7, 2021.

[10] F. Garcia, J. Gonzalez-Bueno, F. Guijarro, and J. Oliver, "Forecasting the environmental, social, and governance rating of firms by using corporate financial performance variables: a rough set approach," Sustainability, vol. 12, no. 8, 2020.

[11] J. Feng, J. W. Goodell, and D. Shen, "Esg rating and stock price crash risk: evidence from China," Finance Research Letters, Article ID 102476, 2021, In press.

[12] B. Auer, "Do socially responsible investment policies add or destroy european stock portfolio value?" Journal of Business Ethics, vol. 135, pp. 1-17, 2014.

[13] A. Kempf and P. Osthoff, "The effect of socially responsible investing on portfolio performance," European Financial Management, vol. 13, no. 5, pp. 908-922, 2007.

[14] M. L. Barnett and R. M. Salomon, "Beyond dichotomy: the curvilinear relationship between social responsibility and financial performance," Strategic Management Journal, vol. 27, no. 11, pp. 1101-1122, 2006.

[15] D. Dominique, T. Hebb, and E. L. H. Touré, "Exploring factors that influence social retail investors decisions: evidence from desjardins fund," Journal of Business Ethics, vol. 134, no. 1, pp. 45-67, 2014.

[16] C. MacKenzie and A. Lewis, "Morals and markets: the case of ethical investing," Business Ethics Quarterly, vol. 9, no. 3, pp. 439-452, 1999.

[17] B. N. Rosen, D. M. Sandler, and D. Shani, "Social issues and socially responsible investment behavior: a preliminary empirical investigation," Journal of Consumer Affairs, vol. 25, no. 2, pp. 221-234, 1991. 
[18] J. Nofsinger and A. Varma, "Socially responsible funds and market crises," Journal of Banking \& Finance, vol. 48, pp. 180-193, 2014.

[19] C. Calvo, C. Ivorra, and V. Liern, "Finding socially responsible portfolios close to conventional ones," International Review of Financial Analysis, vol. 40, pp. 52-63, 2015.

[20] M. von Wallis and C. Klein, "Ethical requirement and financial interest: a literature review on socially responsible investing," Business Research, vol. 8, no. 1, pp. 61-98, 2014.

[21] W. Huang, Y. Nakamori, and S.-Y. Wang, "Forecasting stock market movement direction with support vector machine," Computers \& Operations Research, vol. 32, no. 10, pp. 25132522, 2005.

[22] R. I. Rasel, N. Sultana, and P. Meesad, "An efficient modelling approach for forecasting financial time series data using support vector regression and windowing operators," International Journal of Computational Intelligence Studies, vol. 4, no. 2, p. 134, 2015.

[23] E. Guresen, G. Kayakutlu, and T. U. Daim, "Using artificial neural network models in stock market index prediction," Expert Systems with Applications, vol. 38, no. 8, pp. 1038910397, 2011.

[24] M. Qiu, L. Cheng, and Y. Song, "Application of the Artifical Neural Network in predicting the direction of stock market index," in Proceedings of the 2016 10th International Conference on Complex, Intelligent, and Software Intensive Systems, Fukuoka, Japan, 2016.

[25] M. R. Hassan, "A combination of hidden markov model and fuzzy model for stock market forecasting," Neurocomputing, vol. 72, pp. 3439-3446, 2009.

[26] S. Chakravarty and P. K. Dash, "A pso based integrated functional link net and interval type-2 fuzzy logic system for predicting stock market indices," Applied Soft Computing, vol. 12, no. 2, pp. 931-941, 2012.

[27] O. Lachiheb and M. S. Gouider, "A hierarchical deep neural network design for stock returns prediction," Procedia Computer Science, vol. 126, pp. 264-272, 2018.

[28] R. Singh and S. Srivastava, "Stock prediction using deep learning," Multimedia Tools and Applications, vol. 76, no. 18, pp. 18569-18584, 2017.

[29] W. Zhang, K. Tao, J. Li, Y. Zhu, L. Jing, and H. Cheng, "Modeling and prediction of stock price with convolutional neural network based on blockchain interactive information," Wireless Communications and Mobile Computing, vol. 2020, Article ID 6686181, 10 pages, 2020.

[30] W. Chen, M. Jiang, W.-G. Zhang, and Z. Chen, "A novel graph convolutional feature based convolutional neural network for stock trend prediction," Information Sciences, vol. 556, pp. 67-94, 2021.

[31] T. Fischer and C. Krauss, "Deep learning with long short-term memory networks for financial market predictions," European Journal of Operational Research, vol. 270, no. 2, pp. 654-669, 2017.

[32] K. Chen, Y. Zhou, and F. Dai, "A LSTM-based method for stock returns prediction: a case study of china stock market," in Proceedings of the IEEE International Conference on Big Data, pp. 2823-2824, Santa Clara, CA, USA, 2015.

[33] Y. Zhang, G. Chu, and D. Shen, "The role of investor attention in predicting stock prices: the long short-term memory networks perspective," Finance Research Letters, vol. 38, Article ID 101484, 2021.

[34] F. Zhou, Q. Zhang, D. Sornette, and L. Jiang, "Cascading logistic regression onto gradient boosted decision trees for forecasting and trading stock indices," Applied Soft Computing, vol. 84, 2019.

[35] W. Chen, H. Zhang, M. Kumar Mehlawat, and L. Jia, "Meancvariance portfolio optimization using machine learning-based stock price prediction," Applied Soft Computing, vol. 100, 2021.

[36] X. Li, H. Xie, R. Wang et al., "Empirical analysis: stock market prediction via extreme learning machine," Neural Computing \& Applications, vol. 27, no. 1, pp. 67-78, 2014.

[37] R. Dash and P. Dash, "Stock price index movement classification using a ceflann with extreme learning machine," Power, Communication \& Information Technology Conference IEEE, vol. 10, pp. 22-28, 2015.

[38] Y. Wei, S. Sun, J. Ma, S. Wang, and K. K. Lai, "A decomposition clustering ensemble learning approach for forecasting foreign exchange rates," Journal of Management Science and Engineering, vol. 4, no. 1, pp. 45-54, 2019.

[39] M. Jiang, L. Jia, Z. Chen, and W. Chen, "The two-stage machine learning ensemble models for stock price prediction by combining mode decomposition, extreme learning machine and improved harmony search algorithm," Annals of Operations Research, 2020, In press.

[40] B. Zhu, Y. Feng, D. Gong, S. Jiang, L. Zhao, and N. Cui, "Hybrid particle swarm optimization with extreme learning machine for daily reference evapotranspiration prediction from limited climatic data," Computers and Electronics in Agriculture, vol. 173, Article ID 105430, 2020.

[41] H. Wei, J. Chen, J. Zhu, X. Yang, and H. Chu, "A novel algorithm of nested-elm for predicting blasting vibration," Engineering with Computers, vol. 7, 2020.

[42] P. Mohapatra, S. Chakravarty, and P. K. Dash, "An improved cuckoo search based extreme learning machine for medical data classification," Swarm and Evolutionary Computation, vol. 24, pp. 25-49, 2015.

[43] L. L. Li, J. Sun, M. L. Tseng, and Z. G. Li, "Extreme learning machine optimized by whale optimization algorithm using insulated gate bipolar transistor module aging degree evaluation," Expert Systems with Applications, vol. 127, 2019.

[44] Y. Li and H. Hu, "Influential factor Analysis and projection of industrial CO2 emissions in China based on extreme learning machine improved by genetic algorithm," Polish Journal of Environmental Studies, vol. 29, no. 3, pp. 2259-2271, 2020.

[45] H. Wang, Y. Wang, and Z. Ji, "Simulation of wind power prediction based on improved elm," Journal of System Simulation, vol. 30, pp. 4437-4447, 2018.

[46] Y. Chen, Z.. Wang, Z.. Wang, and L. Xiao, "Research on concentration retrieval of gas ftir spectra by interval extreme learning machine and genetic algorithm," Spectroscopy and Spectral Analysis, vol. 34, pp. 1244-1248, 2014.

[47] N. Zhu, C. Zhu, and E. Ali, "A combined machine learning algorithms and dea method for measuring and predicting the efficiency of Chinese manufacturing listed companies," Journal of Management Science and Engineering, 2020, In press.

[48] I. Oikonomou, E. Platanakis, and C. Sutcliffe, "Socially responsible investment portfolios: does the optimization process matter?" The British Accounting Review, vol. 50, no. 4, pp. 379-401, 2018.

[49] M. Branch, L. R. Goldberg, and P. Hand, "A guide to esg portfolio construction," Journal of Portfolio Management, vol. 45, no. 4, pp. 61-66, 2019.

[50] N. N. Y. Vo, X. He, S. Liu, and G. Xu, "Deep learning for decision making and the optimization of socially responsible investments and portfolio," Decision Support Systems, vol. 124, Article ID 113097, 2019. 
[51] S. Utz, M. Wimmer, and R. E. Steuer, "Tri-criterion modeling for constructing more-sustainable mutual funds," European Journal of Operational Research, vol. 246, no. 1, pp. 331-338, 2015.

[52] C. Calvo, C. Ivorra, and V. Liern, "Fuzzy portfolio selection with non-financial goals: exploring the efficient frontier," Annals of Operations Research, vol. 245, pp. 31-46, 2016.

[53] S. Gasser, M. Rammerstorfer, and K. Weinmayer, "Markowitz revisited: Social portfolio engineering," European Journal of Operational Research, 2017.

[54] A. Bilbao-Terol, M. Arenas-Parra, V. Cañal-Fernández, and C. Bilbao-Terol, "Selection of socially responsible portfolios using hedonic prices," Journal of Business Ethics, vol. 115, no. 3, pp. 515-529, 2013.

[55] G. B. Huang, Y. Qin, and K. Chee, "Extreme learning machine: theory and applications," Neurocomputing, vol. 70, no. 1, pp. 489-501, 2006.

[56] P. Du, J. Wang, W. Yang, and T. Niu, "Container throughput forecasting using a novel hybrid learning method with error correction strategy," Knowledge-Based Systems, vol. 182, p. 10, 2019.

[57] J. Holland, Adaptation in Natural and Artificial Systems, University of Michigan Press, Ann Arbor, MI, USA, 1975.

[58] J.-S. Chen, J.-L. Hou, S.-M. Wu, and Y.-W. Chang-Chien, "Constructing investment strategy portfolios by combination genetic algorithms," Expert Systems with Applications, vol. 36, no. 2, pp. 3824-3828, 2009.

[59] F. Amini and G. Hu, "A two-layer feature selection method using genetic algorithm and elastic net," Expert Systems with Applications, vol. 166, Article ID 114072, 2021.

[60] E. Tekeli, N. özbay, and S. Kaçiranlar, "Implementation and validation of new optimization methods by genetic algorithm for two-parameter ridge estimator," Concurrency and Computation: Practice and Experience, vol. 33, no. 9, p. 5, 2021.

[61] S. S. Moghaddasi and N. Faraji, "A hybrid algorithm based on particle filter and genetic algorithm for target tracking," Expert Systems with Applications, vol. 147, Article ID 113188, 2020.

[62] B. Chen, J. Zhong, and Y. Chen, "A hybrid approach for portfolio selection with higher-order moments: empirical evidence from shanghai stock exchange," Expert Systems with Applications, vol. 145, Article ID 113104, 2020.

[63] E. C. Brown and R. T. Sumichrast, "Evaluating performance advantages of grouping genetic algorithms," Engineering Applications of Artificial Intelligence, vol. 18, no. 1, pp. 1-12, 2005.

[64] Y. Zheng, M. Huang, Yi Lu, and W. Li, "Fractional stochastic resonance multi-parameter adaptive optimization algorithm based on genetic algorithm," Neural Computing \& Applications, vol. 32, no. 22, p. 11, 2020.

[65] H. Park, D. Son, B. Koo, and B. Jeong, "Waiting strategy for the vehicle routing problem with simultaneous pickup and delivery using genetic algorithm," Expert Systems with Applications, vol. 165, Article ID 113959, 2021.

[66] Y. Chen and X. Wang, "A hybrid stock trading system using genetic network programming and mean conditional valueat-risk," European Journal of Operational Research, vol. 240, no. 3, pp. 861-871, 2015.

[67] W. Wang, W. Li, N. Zhang, and K. Liu, "Portfolio formation with preselection using deep learning from long-term financial data," Expert Systems with Application, vol. 143, pp. 113042.1-113042.17, 2020.

[68] Z. Mohamed, D. Mohamad, and O. Samat, "Maximizing portfolio diversification benefit via extended mean-variance model," in Proceedings of the IEEE Symposium on Industrial Electronics and Applications, p. 10, Penang, Malaysia, 2010.

[69] G. Y. N. Tang, "How efficient is naive portfolio diversification? an educational note," Omega, vol. 32, no. 2, pp. 155-160, 2004.

[70] S. Sun, S. Wang, and Y. Wei, "A new ensemble deep learning approach for exchange rates forecasting and trading," Advanced Engineering Informatics, vol. 46, Article ID 101160, 2020. 